\title{
Thermal diffusivity evaluation of Thermal Barrier Coatings by photothermal and thermographic techniques
}

Federico Cernuschi, Angelamaria Figari, Lorenzo Lorenzoni, Davide Robba.

Cesi spa

Via Rubattino, 54

20134 Milano

Italy

Tel. +39-02 21254577

e-mail cernuschi@cesi.it

Keywords: thermal diffusivity, thermography, coating, NDE.

\begin{abstract}
In order to improve the thermal insulation of the gas turbine hot path components, porous thermal barrier coatings (TBCs) are widely applied. The monitoring and the characterisation of the thermal properties of TBC are essential since TBC performances strongly depend on the microstructure as well as on the service conditions. In fact, the prolonged exposure to high temperature can promote shrinkage phenomena within the TBC, which make the coating less strain tolerant and more heat conductive: this leads to a drastic reduction of the functionality of this porous ceramic protective top layer.

To face the problem of estimating non destructively the evolution of the functional effectiveness of TBC deposited onto components like blades and vanes, we have performed the study of the evolution of the thermal diffusivity as a function of operating conditions, as a first step. In particular the thermal diffusivity of free-standing TBCs samples aged at different temperatures and for different times has been measured by using the laser flash technique. Collecting all the
\end{abstract}


data, a curve of normalised thermal diffusivity of TBC as a function of the Larson-Miller parameter - which conglobates time and temperature - has been obtained. This curve describes the evolution of the thermal diffusivity but it gives also indications about the functional life of the TBC. A NDE technique able to measure the TBC thermal diffusivity directly onto the components appears useful for monitoring the evolution of the serviced TBC and getting indications about the residual life of the ceramic coating.

In this work, a thermographic non destructive technique for evaluating the thermal diffusivity of TBC directly onto components is presented. In particular, the results of theoretical modelling and experimental activities are reported in the frame of developing two different single side thermographic techniques for measuring the in-plane and the through-the-thickness thermal diffusivity respectively. 